\title{
Full Prevalence Of Gestational Diabetes In Urban Women In Blantyre, Malawi: A Cross Sectional Study And Comparison Of Who And IADPSG Criteria
}

Tamara Joy Phiri ( $\nabla$ tamaraphiri@gmail.com )

Queen Elizabeth Central Hospital

Marianne Kasiya

Queen Elizabeth Central Hospital, Blantyre, Malawi

Theresa J Allain

Department of Internal Medicine, College of Medicine, Blantyre, Malawi

Research article

Keywords: gestational diabetes mellitus, diabetes mellitus, non-communicable diseases, pregnancy, subSaharan Africa, Malawi

Posted Date: August 23rd, 2019

DOl: https://doi.org/10.21203/rs.2.13491/v1

License: (c) (i) This work is licensed under a Creative Commons Attribution 4.0 International License. Read Full License 


\section{Abstract}

Aims This study aimed to establish the prevalence of gestational diabetes mellitus (GDM) among urban women in Blantyre where the prevalence was previously unknown. A comparison was made between the prevalence determined using WHO criteria and that using the recent International Association of Diabetes in Pregnancy Study Group (IADPSG) criteria. Methods 2274 consecutive women presenting to five antenatal clinic sites in Blantyre had a screening random blood glucose (RBG). Of these, a subsample of 250 women were randomly selected for an oral glucose tolerance test (OGTT). Logistic regression was used to quantify the association between various exposure variables and prevalence of diabetes. Results Mean age was 25 years (range $14-43$ years), $66 \%$ were in the third trimester. Mean RBG was $5.1 \mathrm{mmol} / \mathrm{L}$ (range 2.4 - 10.6). Overall prevalence of GDM was $1.6 \%$ and $24 \%$ using the WHO and IADPSG criteria respectively. GDM was associated with older maternal age, high parity and attendance at government antenatal clinics but not with MUAC, BMI, positive family history of diabetes or previous poor neonatal outcome. There was no correlation between random blood glucose and gestational diabetes diagnosed on the oral glucose tolerance test. Conclusions The prevalence of GDM in Blantyre using WHO criteria was low in the predominantly young population that was screened. A much higher proportion had GDM based on the IADPSG criteria and these may warrant long term follow up. GDM was not associated with some previously described risk factors for GDM suggesting a different risk factor profile compared to high income countries.

\section{Background}

Gestational diabetes mellitus (GDM) is defined as any degree of glucose intolerance with onset or first recognition during pregnancy. [1] It is associated with an increased risk of pre-eclampsia, macrosomia and risk of the mother developing Type 2 diabetes after pregnancy. Infants born to mothers with GDM are at an increased risk for birth trauma and neonatal metabolic complications including hypoglycemia, hypocalcemia and hyperbilirubinaemia. [2, 3]. Risk factors for GDM in developed countries include advanced maternal age, obesity and a family history of diabetes. [4]

Few studies have been done in Africa on the prevalence of GDM. Comparisons between the studies are limited by heterogeneity of the study populations, small sample sizes and variable diagnostic criteria used. These studies reported a prevalence of 3.8\% in South Africa (1989), [5] 0\% in Tanzania (1990), [6] $11 \%$ in Nigeria (1997), [7] 3.7\% in Ethiopia (1997) [8] and 3.8\% in South Africa (2007). [9] All five studies used World Health Organization (WHO) criteria for GDM diagnosis.

In Malawi, the nationwide WHO STEPWise Approach to Surveillance (STEPS) survey in 2009 found that $5.6 \%$ of adult Malawians had diabetes, the majority of which was undiagnosed [10]. There are no studies on the prevalence of gestational diabetes in Malawi. 
There has been a universal lack of consensus on screening and diagnosis of GDM with regards to the impact of screening on outcomes, appropriate individuals to screen, optimal screening time, the appropriate screening tool and appropriate diagnostic criteria. This has resulted in several revisions of diagnostic criteria by various groups. Two large studies that have influenced the interpretation of diagnostic criteria in recent times are the 2005 Australian Carbohydrate Intolerance Study in Pregnancy (ACHOIS) [11] which demonstrated that mild forms of hyperglycemia, below those diagnostic of GDM, are associated with poor perinatal outcomes and the 2008 Hyperglycaemia and Pregnancy Outcomes (HAPO) study [12] which showed a linear association between maternal hyperglycemia and adverse events including macrosomia, pre-eclampsia, caesarean section rates and neonatal hypoglycemia with no clear cut-off above which these adverse events occurred. Following the HAPO study, the IADPSG recommended new criteria for the diagnosis of GDM with a fasting plasma glucose cut-off much lower than the WHO criteria. [13] diagnosed as having GDM using IADPSG criteria as compared to the WHO criteria. There is varied opinion as to whether IADPSG criteria universally translate into improved outcomes particularly when applied to a population that is different from that in the HAPO study. [14 -16]

Some guidelines favour selective screening of women with known risk factors for GDM in order to avoid unnecessary screening of low risk women. Whether the traditional risk factors as described in studies in high income countries are applicable to and predict GDM in sub Saharan Africa has not been explored. Establishing a risk factor profile for women with GDM to be prioritized for screening is essential, particularly in a low resource setting like Malawi where routine screening of all pregnant women is not feasible. Random blood glucose, fasting blood glucose and the $50 \mathrm{~g}$ oral glucose tolerance test (OGTT) have all been used in studies as screening tests. [17] Finger prick random blood glucose, though inferior to formal laboratory glucose tests, is a feasible screening option in Malawi where the majority of the population has limited access to formal blood glucose tests.

This study aimed to establish the prevalence and risk factors for GDM amongst urban women in Blantyre, Malawi. Additionally, we compared the prevalence using the WHO and IADPSG criteria and assessed the use of random blood glucose as a potential screening tool for GDM in this population.

\section{Methods}

Blantyre is the main commercial city in Southern Malawi, with an estimated population of 1.1 million. [18] Queen Elizabeth Central Hospital (QECH) is the main government funded tertiary referral centre.

Chilomoni and Limbe health centres are government funded primary care facilities in Blantyre with an average antenatal clinic attendance of 100 women per day. Mwaiwathu and Blantyre Adventist hospitals are the two main private hospitals in Blantyre. 
In this cross-sectional study, consecutive women presenting at any gestational age to QECH, Chilomoni and Limbe antenatal clinics (ANCs) between $1^{\text {st }}$ June and 30 September 2012 and at Mwaiwathu and BAH private ANCs between February and April 2013 were asked to participate in the study. Recruitment was restricted to women of Malawian origin residing in Blantyre during the study period.

Consenting women had a capillary random blood glucose (RBG) done at the clinic site with a finger prick test and an SDCheck ${ }^{\circledR}$ Glucometer (SD Standard Diagnostics Inc. Hagal-dong, Korea). A sub-sample of 200 women from the government ANCs and 50 women from the private ANCs were randomly selected for an OGTT by selecting every fifth woman that was recruited. Gestational age was calculated from the last normal menstrual period.

For RBG, a sample size of 614 was initially calculated in order to detect hyperglycaemia at an estimated prevalence of 2-3\% (suggested by local Blantyre obstetricians from observation) but the sample size was subsequently increased after detecting a high proportion of normal RBGs when recruitment began. Furthermore, the test could easily be administered to large numbers of women attending the facilities within a short period of time. The sample size of 250 for OGTTs was limited by available resources to perform OGTTs.

All OGTTs were done at QECH laboratory and plasma glucose was analyzed using an automatic analyzer (KeyLab BPC BioSed ${ }^{\circledR}$ Rome, Italy). OGTTs were done following the 1999 WHO guidelines with each participant having a fasting plasma glucose done and then given $75 \mathrm{~g}$ of anhydrous glucose dissolved in $200 \mathrm{mls}$ of water to drink. Plasma glucose was re-checked 2 hours after taking the glucose solution.

Using the WHO criteria, [19] GDM was defined as a fasting plasma glucose $7.0 \mathrm{mmol} / \mathrm{L}$ or a 2-hour plasma glucose $11.1 \mathrm{mmol} / \mathrm{L}$. Using the modified IADPSG criteria, [13] GDM was defined as fasting plasma glucose $\geq 5.1 \mathrm{mmol} / \mathrm{L}$ or 2 hours plasma glucose $8.5 \mathrm{mmol} / \mathrm{L}$.

Blood pressure (BP), weight, height and mid-upper arm circumference (MUAC) were recorded on recruitment. BP was measured with an Omron ${ }^{\circledR}$ digital BP machine (Omron Healthcare Worldwide, Kyoto, Japan). Weight was measured using a digital scale. Where previously documented in the woman's health records, the pre-pregnancy weight was noted. The majority of the women did not have a documented prepregnancy weight or height and pre-pregnancy BMI could not be calculated. 
Mid upper arm circumference (MUAC) was used to assess nutritional status as a single BMI in pregnancy is not an accurate measure because of the additional weight gain from pregnancy. MUAC has been shown to correlate well with pre-pregnancy BMI where a pre-pregnancy BMI of 18.5, 25-30 and > 30 roughly correlates with a MUAC of $23 \mathrm{~cm}, 28-32 \mathrm{~cm}$ and $>32 \mathrm{~cm}$ respectively. [20, 21] BMI in pregnancy at the time of recruitment was nevertheless calculated (weight $(\mathrm{kg}) /$ height $(\mathrm{m})^{2}$ ).

Patients diagnosed with GDM or hypertension were referred to the QECH, Mwaiwathu and BAH specialist diabetes clinics for follow up and management.

\section{Statistical Analysis}

Means and percentages were used to explore the distribution of risk factors between government and private ANCs. The relationship between prevalence and risk factors was first explored through univariate analyses. T-test comparing women with GDM to those without GDM were used to assess if any of the continuous risk factors were associated with prevalence. To adjust for possible simultaneous confounding of the risk factors, a multivariate logistic regression was fitted. The stepwise model selection method was employed to come up with the final model which included the following risk factors: type of hospital, age, parity, MUAC and history of macrosomia for the WHO criteria. Similarly, a multivariate linear regression was used to assess the relationship between random blood glucose and the risk factors. The final selected model included hospital type, BMI and history of macrosomia.

SAS software version 9.3 (SAS Institute, North Carolina State University) was used for analysis and all inferences were made at 0.05 significance level.

\section{Results}

\section{General Demographics}

All participants were recruited from urban Blantyre. In total, 2350 consecutive women presenting to the above-named facilities were asked to participate in the study. Of these 2274 consented and proceeded to have a random blood glucose screen while 76 declined consent. Of the 2274, 52 had missing data and were excluded from the analysis on demographics and random blood glucose. 250 women were randomly selected for an OGTT from the 2274 that had a random blood glucose screen. 57 did not turn up for the test and 193 women were included in the final analysis on OGTTs.

Table 1 compares the demographic characteristics of women in government and private ANCs. 
The study population was predominantly young with an average age of 25.8 years $\left(25^{\text {th }}, 50^{\text {th }}, 75^{\text {th }}\right.$ percentiles: $22,25,30) .6 \%$ of the women were above 35 years of age. $66.4 \%$ of the women were in the third trimester. $24 \%$ of the women were between 24 - and 28 -weeks gestation.

Women at government funded facilities were younger, of higher parity and gravidity, had a lower pregnancy BMI and were more likely to be HIV positive. Based on MUAC, $9 \%$ of the women were overweight (MUAC 28-31 cm) and $1 \%$ were obese (MUAC $\geq 32 \mathrm{~cm}$ ). There was no difference in the average MUAC between government and private ANCs. When the BMI in pregnancy was calculated, half of the women had a normal BMI (average BMI 26; 50th percentile 25). There was a non-significant difference in the BMI between women from government and private ANCs.

3.3\% had hypertension but this was not explored further to determine whether this was pre-eclampsia or pre-existing hypertension. $10.7 \%$ of the women had HIV and of these, $61 \%$ had documented records of being on anti-retroviral therapy.

\section{Blood Glucose and GDM Prevalence}

Tables 2 and 3 show RBG and OGTT results. Only 3 women (0.1\%) had an RBG above $11.1 \mathrm{mmol} / \mathrm{L} .12$ women $(0.5 \%)$ were hypoglycaemic. There was a significant association between RBG and attending government ANCs and BMI.

Based on the OGTTs, the overall prevalence of GDM was $1.6 \%(n=5)$ and $24.8 \%(n=65)$ by WHO and IADPSG criteria respectively. The simple Kappa coefficient was calculated to determine correlation between GDM diagnosed by the WHO and GDM diagnosed by IADPSG criteria and was found to be 0.597 (a coefficient of zero being no correlation and a coefficient of one being a strong correlation).

\section{Risk Factors for GDM}

Tables 4 shows risk factors associated with GDM5.3\% of the women reported to have previously had macrosomic babies (birth weight $>4 \mathrm{~kg}$ ). This could not be confirmed as the majority did not have written records of birth weights from previous pregnancies and their responses were based on recall. $7 \%$ reported to have first degree family relations with diabetes and $19.2 \%$ reported to have had miscarriages or stillbirths in previous pregnancies.

Attending government ANCs, age and parity were associated with having GDM ( $p$ value $<0.05$ ). The risk of having GDM was higher in government ANCs compared to private ANCs and this increased with age and parity. A family history of diabetes mellitus, previous miscarriage/still birth, BMI, being HIV positive and hypertension were not associated with GDM ( $p$ values >0.05).

\section{Outcomes}

$71 \%$ of the women diagnosed with GDM were lost to follow up post-delivery and complete outcome data was only available for 18 women. There were 4 miscarriages, 4 women that had Caesarean Section and 2 
babies with macrosomia. Data on follow up for diabetes at 6 weeks postpartum in particular was missing as this was collected telephonically and some of the women could not be reached.

\section{Discussion}

This study showed that the prevalence of gestational diabetes mellitus in Blantyre is low. It also showed a wide discrepancy in the prevalence when IADPSG criteria are used compared to the WHO criteria with a 12 -fold increase in the prevalence when the IADPSG criteria are used. To our knowledge this is the first description of the prevalence of gestational diabetes in the Malawian population.

The HAPO study, with an average BMI of 27 among its participants, showed a direct correlation between obesity and poor outcomes. [14] Our study population however, being largely young with few obese women ( $1 \%$ based on MUAC), was different from that described in other studies of risk factors for GDM. Furthermore, half of the women had normal BMI during pregnancy.

In the nationwide WHO Steps survey [10] , the prevalence of overweight and obesity among Malawian women was $16 \%$ and $2 \%$ respectively. The age of the women screened was $25-64$ years, but the majority of the women screened were young as $46 \%$ of the women were between ages $25-34$. Our GDM study similarly screened a young population of women and the prevalence of overweight and obesity were $9 \%$ and $1 \%$ respectively. From both studies, obesity appears to be rare amongst Malawian women.

In another 2007 study of 620 patients attending the adult diabetic clinic at QECH, the average BMI in Type 2 DM patients was 28.7. [24] These observations suggest that obesity may not be the main driver for the diabetes epidemic in Malawi and that other factors such as genetics, low birth weight and stunting may play a larger role.

\section{Risk Factors for Gestational Diabetes}

Advanced maternal age, high parity and attending government ANCs were associated with GDM, the older women being more likely to have high parity than the young consistent with traditional risk factors for GDM. Other known risk factors for GDM such as a family history of diabetes, a history of macrosomia or previous miscarriages or stillbirths or MUAC were not associated with GDM. As observed in the STEPS survey, the majority of diabetes in the population is undiagnosed; as such a negative family history of diabetes may in part be a reflection of this. The overall picture however highlights the fact that risk factors for developing GDM may be population specific and there may be genetic variability inherent to the population to explain such differences. This raises a cause for exploring population specific risk factors other than those stated in the WHO guidelines or those from high income countries.

Women attending private hospitals are generally perceived as having a higher socioeconomic status and more likely to adopt a diet rich in refined foods and a sedentary lifestyle than their counterparts. By including private ANCs, we anticipated to show that this group would tend to be more obese and have a 
higher risk of developing GDM. Our findings though were contrary to this expectation as there was no difference in terms of nutritional status between women from government facilities and those from private hospitals. Furthermore, women at private ANCs were less likely to have GDM than those in government hospitals. Diet differences between the two groups were not explored in particular but it appears that the risk that may be conferred by sedentary habits or a westernized diet may be balanced by better health seeking behavior and ready access to screening and diagnostic services in the private hospitals.

\section{GDM Screening}

Random blood glucose measurements were largely normal as only 3 women had RBG $>11.1 \mathrm{mmol} / \mathrm{L}$ and $75 \%$ of the study population had an RBG below $5.5 \mathrm{mmol} / \mathrm{L}$. Other than the RBG being an insensitive screening tool, it was also observed on random questioning that many of the women at the health centres had not eaten for some time before the measurement particularly those that had to leave their homes early in the morning to attend clinic on time. Their results may reflect a fasting rather than random blood glucose and may explain the large proportion of women with normal RBG. There was no correlation between the random blood glucose and gestational diabetes diagnosed by OGTT or risk factors for diabetes. Random blood glucose may therefore not be a sensitive screening tool or used as a proxy for OGTTs in this population.

\section{GDM Diagnosis}

The prevalence of GDM using WHO criteria of $1.6 \%$ was lower than that described in other African studies using the same diagnostic criteria (3.8\% - South Africa and 3.8\% in Ethiopia) [11,12] but comparable with what was expected by local obstetricians who estimated prevalence between 2-3\% amongst women attending antenatal clinics (B. Makanani personal communication). GDM was rare even amongst those with traditional risk factors for GDM suggesting there may be a unique environmental or genetic influence on risk factors for GDM in this population.

Using IADPSG criteria, the prevalence of GDM was 12 times higher compared to the WHO criteria and interestingly showed a higher prevalence in government ANCs compared to private ANCs.

We anticipated to find a higher prevalence of GDM using IADPSG criteria as compared to WHO criteria as has been described in other studies. There are no other published studies from African populations for comparison. Many studies have compared prevalence using the two criteria with some finding the two to be comparable. [16] The decision to change the criteria depends on performing careful cost analysis and weighing the risk benefit ratio particularly in a population that is different from the HAPO population performance in a non HAPO population thought to be lower. [22] In a low income setting, particularly where maternal obesity which was shown to be an independent risk factor for poor perinatal outcomes (Catalano) is rare, priority should probably be placed on treating those diagnosed with GDM based on WHO criteria. 
There was a large loss to follow up amongst the women diagnosed with GDM which precludes definitive conclusions on outcome. The causes of the four miscarriages among the women diagnosed with GDM were not explored further.

\section{Limitations}

The study had several limitations. The study population being urban may have been unrepresentative as it excludes older women in rural settings likely to have risk factors for GDM. Older women and multiparous women are less likely to attend formal antenatal care clinics. Family history of DM was likely underreported as most diabetes in Malawi is still undiagnosed. Loss to follow up precluded making meaningful conclusions on outcomes on the already small population of women diagnosed with GDM. Digital instruments used for measuring anthropometric and biochemical data including glucometers, blood pressure machines and the weight scale though readily accessible for use in the practical sense are not always standardized and may be inappropriately calibrated which affects quality and reproducibility of data collected.

Being descriptive, definite causal relationships cannot be established. A larger prospective study with OGTTs performed on all women, exploring risk factors for GDM and comparing outcomes between the WHO and IADPSG criteria would reflect better on the usefulness of diagnosing GDM in this population.

\section{Conclusions}

In conclusion, using WHO criteria, GDM is relatively uncommon in women presenting to antenatal clinics even amongst those with traditional risk factors for GDM in Blantyre. This low prevalence has been demonstrated in other sub-Saharan countries and we anticipate that the prevalence would be similar in the Malawian population in general. The implications of the higher prevalence found when IADPSG criteria are used remain to be explored.

Increasing age, parity and being at public hospitals are associated with GDM in this population. Alternative risk factors other than the traditional known risk factors need to be explored. Maintaining optimal weight should be encouraged as this is the single modifiable risk factor for GDM that has been identified in this study. Should screening for GDM be performed, random blood glucose is not a sensitive screening tool and risk factor-based screening may be more feasible and cost effective.

\section{Abbreviations}


HIV Human Immunodeficiency Virus

IADPSG International Association of the Diabetes and Pregnancy Study Group

MUAC Mid-Upper Arm Circumference

OGTT

Oral Glucose Tolerance Test

QECH

Queen Elizabeth Central Hospital

RBG

Random Blood Glucose

WHO

World Health Organization

\section{Declarations}

\section{Ethics, Consent and Approvals}

Ethical approval for the study was obtained from the Malawi College of Medicine Research and Ethics Committee (reference number P02 12 1170). Each participant provided written consent. For participants that could not read or write, the consent form was read out to them by a research assistant and the participant gave verbal consent and put their fingerprint on the consent form to acknowledge their voluntary participation in the study. The consent form was available in English and vernacular and had been approved by the College of Medicine Research and Ethics Committee prior to commencement of the study.

\section{Funding}

This study was done with funding from the World Diabetes Foundation grant number WDF 09-451. The funder had no role in study design, data collection, analysis, interpretation or in writing the manuscript.

\section{Competing Interests}

The authors declare that they have no competing interests

\section{Author Contributions}


TA conceived the original idea and supervised the project. TP carried out the study, analyzed and interpreted data and prepared the manuscript. MK recruited study participants, collected and compiled data. All authors read and approved the final manuscript.

\section{Availability of Data and Materials}

All data generated or analysed during this study are included in this published article and its supplementary information files

\section{Consent For Publication}

Not applicable

\section{Acknowledgements}

The authors thank the antenatal clinic patients, clinicians and nurses at Queen Elizabeth Central Hospital, Chilomoni Health Centre, Limbe Health Centre, Mwaiwathu and Blantyre Adventist hospitals, Mr Henry Feluzi, Mr Mavuto Mukaka and Miss Elasma Milanzi.

\section{References}

1. World Health Organization. Guidelines for the prevention, management and care of diabetes mellitus. 2006, EMRO Technical Publications Series 32

2. Setji TL, Brown AJ, Feinglos MN. Gestational diabetes. Clinical Diabetes. 2005; 23:17-24

3. Kuhl C. Etiology and pathogenesis of gestational diabetes. Diabetes Care. 1998; 21 (Supplement 2): B19-B26

4. Ferrara A. Increasing Prevalence of Gestational Diabetes Mellitus A public health perspective. Diabetes Care. 2007; 30 (Supplement 2): S141 - S146 
5. Ranchod HA, Vaughan JE, Jarvis P. Incidence of gestational diabetes at Northdale Hospital, Pietermaritzburg. S Afr Med J 1991;80: 14 -16

6. Swai AB, Kitange HM, McLarty DG, Kilima PM, Masuki G, Mtinangi BL et al. No deterioration of oral glucose tolerance during pregnancy in rural Tanzania. Diabet Med. 1991; 8: 254-257

7. Olarinoye JK, Ohwovoriole AE, Ajayi GO. Diagnosis of gestational diabetes mellitus in Nigerian pregnant women - Comparison between $75 \mathrm{~g}$ and $100 \mathrm{~g}$ oral glucose tolerance tests. West Afr J Med. 2004; 23:198 - 201

8. Seyoum B, Kiros K, Haileselase T, Leole A. Prevalence of gestational diabetes mellitus in rural pregnant mothers in northern Ethiopia. Diabetes Research and Clinical Practice. 1999; 46: 247-251

9. Mamabolo RL, Alberts M, Levitt NS, Delemarre-van de Waal HA, Steyn NP. Prevalence of gestational diabetes mellitus and the effect of weight on measures of insulin secretion and insulin resistance in third-trimester pregnant rural women residing in the Central Region of Limpopo Province, South Africa. Med. 2007; 24, 233-239

10. Msyamboza KP, Mvula C, Kathyola D. Prevalence and correlates of diabetes mellitus

in Malawi: population-based national NCD STEPS survey. BMC Endocrine Disorders. 2014, 14:41

11. Crowther, C.A., Hiller, J.E., Moss, J.R., McPhee, A.J., Jeffries, W.S., Robinson, J.S.; Australian Carbohydrate Intolerance Study in Pregnant Women (ACHOIS) Trial Group. Effect of treatment of gestational diabetes mellitus on pregnancy outcomes. N Engl J Med. 2005; 352: 2477-2486

12. HAPO Study Cooperative Research Group. Hyperglycemia and adverse pregnancy

outcomes. N Engl J Med. 2008; 358: 1991-2002 
13. Metzger BE, Gabbe SG, Persson B, Buchanan TA, Catalano PA, Damm P et al.

International association of diabetes and pregnancy study groups recommendations on the diagnosis and classification of hyperglycemia in pregnancy. Diabetes Care. 2010;33:676-682

14. Cundy T, Ackermann E, Ryan EA. Gestational diabetes: new criteria may triple the prevalence but effect on outcomes is unclear. 2014; 348

15. Reece EA, Thomas M. The diagnostic criteria for gestational diabetes: to change or not to change? American Journal of Obstetrics \& 2013; 255-259

16. Wendland EM, Torloni MR, Falavigna M, Trujillo J, Dode MA, Campos MA et al. Gestational diabetes and pregnancy outcomes - a systematic review of the World Health Organization (WHO) and the International Association of Diabetes in Pregnancy Study Groups (IADPSG) diagnostic criteria. BMC Pregnancy and Childbirth. 2012, 12:23

17. Tieu J, Middleton P, McPhee AJ, Crowther CA. Screening and subsequent management for gestational diabetes for improving maternal and infant health. Cochrane Database of Systematic Reviews. 2010; Issue 7

18. Malawi National Statistical Office 2008 Population and Housing Census Results Available: http://www.nsomalawi.mw/index.php/2008-population-and-housing-census/107-2008-populationand-housing-census-results.html

19. World Health Organization. Diagnostic Criteria and Classification of Hyperglycaemia First Detected in Pregnancy. 2013

20. Tang AM, Dong K, Deitchler M, Chung M, Maalouf-Manasseh Z, Tumilowicz A et al.Use of Cutoffs for Mid-Upper Arm Circumference (MUAC) as an Indicator or Predictor of Nutritional and Health-Related Outcomes in Adolescents and Adults: A Systematic Review. Washington, DC: FHI 360/FANTA. 2013 
21. Okereke CE, Anyaehie UB, Dim CC, lyare EE, Nwagha UI.Evaluation of some anthropometric indices for the diagnosis of obesity in pregnancy in Nigeria: a cross-sectional study. African Health Sciences. 2013; 13(4): $1034-1040$

22. Cohen DB, Allain TJ, Glover S, Chimbayo D, Dzamalala H, Hofland HW et al. A Survey of the Management, Control, and Complications of Diabetes Mellitus in Patients Attending a Diabetes Clinic in Blantyre, Malawi, an Area of High HIV Prevalence. Am. J. Trop. Med. Hyg., 83(3), 2010, 575-581

23. Leary J, Pettitt DJ, Jovanovic L. Gestational diabetes guidelines in a HAPO world. Best Pract Res Clin Endocrinol Metab. 2010;24(4):673

\section{Tables}

Table 1: Comparison of Characteristics Between Government and Private Antenatal Clinics (ANCs).

Characteristic

Government ANCs $(n=2044)$

Mean $\pm S D$

Age (years)

$25.8 \pm 0.25$

$<0.0001$

Gestational Age (weeks) $\quad 26.9 \pm 0.36$

3.2

Parity

0.13

Gravidity

0.12

BMI in Pregnancy

0.8

MUAC (cm)

\subsection{8}

$$
1.2 \pm 0.05
$$$$
<0.0001
$$

$$
2.5 \pm 0.05
$$$$
<0.0001
$$

Private ANCs $(\mathrm{n}=178) \quad p$ value

Mean $\pm S D$ 
Hypertension

HIV

(8.4)

DM Family History

(24.1)
$97(4.7)$

$$
0.6574
$$

205 (10.0)

$$
0.01
$$

$15(0.7)$

43

0.1573

Previous Miscarriage/Stillbirth 361 (17.6)

$41(23.0)$

0.308

Table 2 : Risk Factors Associated with Increasing_Random Blood Glucose

Variable Parameter Estimate Standard Error t value p value

Government -15.50589

4.763

$-3.26$

0.0013

Hospital

BMI

1.09278

0.37628

2.90

0.0041

Macrosomia $\quad-22.14294$

12.98251

$-1.71$

0.0898

\section{Table 3: Comparison of Random Blood Glucose, Fasting Glucose and GDM Prevalence by OGTTs in Government} and Private ANCs

\begin{tabular}{lrr} 
Government & \multicolumn{1}{c}{ Private } & Overall \\
Mean \pm SD & Mean $\pm S D$ & Mean $\pm S D$ \\
RBG (g/dL) & $94.4 \pm 20$ & $107 \pm 24$ \\
& $94 \pm 21$ & \\
Fasting Glucose $(\mathrm{g} / \mathrm{dL})$ & $84 \pm 16$ & $70 \pm 16$ \\
$81 \pm 19$ &
\end{tabular}




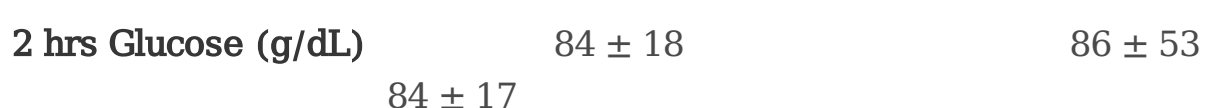

$$
84 \pm 17
$$

CI]

$\%[95 \% C I]$

$\%[95 \% C I]$

$\%[95 \%$

$O R^{*}[95 \% \mathrm{CI}]$

GDM (WHO)

1.4 [0.04-5.5]

$0.04[0-1]$

$1.6 \quad[0.3$

$-4]$

3.5 [0.08 -8.1]

GDM (IADPSG)

31.7 [24.6-39.8]

7.8 [3-19.1]

24.8 [19 -32]

\section{5 [1.9 -16]}

*GDM Prevalence Odds Ratio Government ANCs/Private ANCs

Table 4: Risk Factors Associated With GDM by WHO Criteria

\begin{tabular}{lcccc} 
Parameter & \multicolumn{2}{c}{ Estimate Standard Error } & $\begin{array}{c}\text { Wald } \\
\text { Chi-Square }\end{array}$ & P value \\
& & & & \\
& & & 12.2527 & 0.0005 \\
\hline Government Hospitals & $\mathbf{2 . 0 8 6 0}$ & 0.5959 & & \\
\hline Age & & & 5.2690 & 0.0217 \\
\hline Parity & $\mathbf{0 . 0 9 7 3}$ & 0.0424 & 6.8541 & 0.0088 \\
\hline MUAC & $-\mathbf{0 . 6 1 6 0}$ & 0.2353 & 1.9534 & 0.1622 \\
\hline Previous Macrosomia & $\mathbf{- 1 . 8 4 1 6}$ & 0.9789 & 3.5391 & 0.0599
\end{tabular}

\section{Figures}




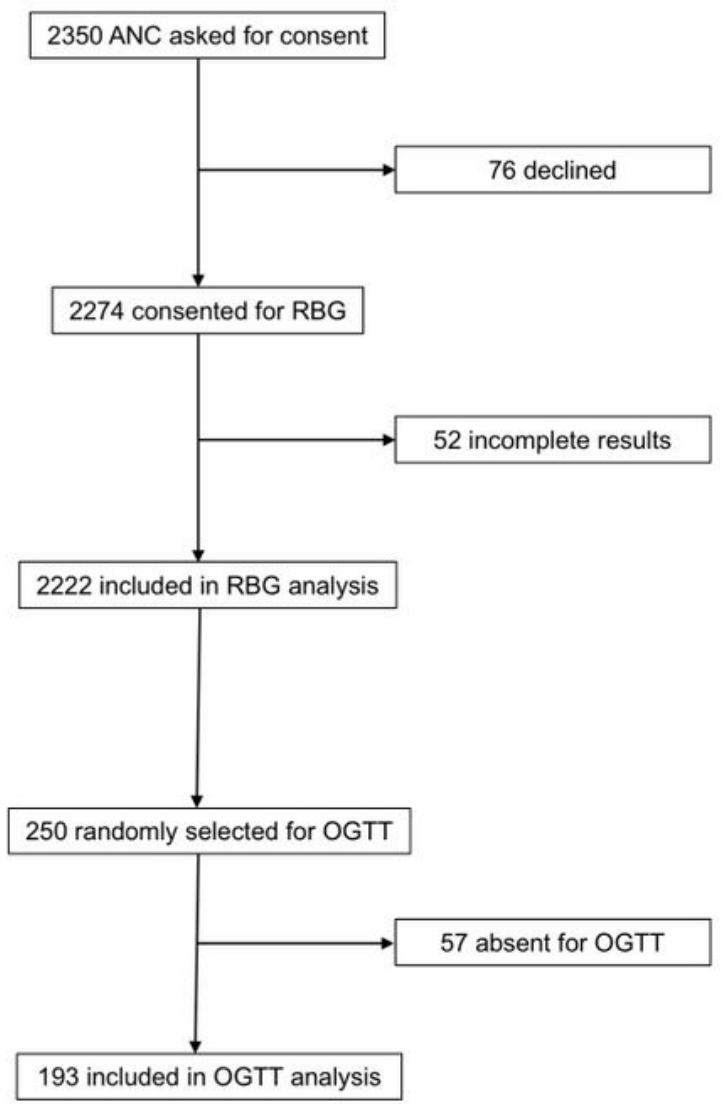

\section{Figure 1}

\section{Supplementary Files}

This is a list of supplementary files associated with this preprint. Click to download.

- CompletedSTROBEchecklistcrosssectional.doc 
- OGTTsAllWithoutldentifiersBMC.xIsx

- BAHandMwaiwathuWithoutldentifiersBMC.xIsx 